\title{
Flight Dynamic Stability of a Flapping Wing Micro Air Vehicle in Hover
}

\author{
Weihua $\mathrm{Su}^{1}$ and Carlos E. S. Cesnik ${ }^{2}$ \\ University of Michigan, Ann Arbor, MI, 48109-2140
}

\begin{abstract}
This paper discusses a methodology of analyzing the flight dynamic stability of a flapping wing Micro Air Vehicle (MAV) in hover. The flexible flapping wings are modeled by a strain-based geometrically nonlinear beam formulation, coupled with an empirical aerodynamic formulation for load calculation on the wings surfaces. Wing flapping kinematics is described using a set of Euler angles. Nonlinear equations of motion for the body frame attached to the vehicle are used to complete the coupled aeroelastic and flight dynamic formulation. All these formulations are implemented in an integrated numerical framework. To evaluate the flight dynamic stability of the hovering flapping wing MAV, the coupled nonlinear governing equations are linearized, and the transition matrix over a wing flapping cycle is determined. By taking advantage of the periodic hovering condition, the stability analysis is performed based on the transition matrix in the Floquet theory. Longitudinal and lateral stabilities of a flapping wing MAV in hover is explored with the impact of different wing rigidity and inertia.
\end{abstract}

\section{Introduction}

$\mathrm{T}$ HE rise and growth of interest in flapping wing Micro Air Vehicles (MAVs) have been stimulated by the long history of natural flight studies. These vehicles have the potential to fly in constrained spaces and to gather information for environmental monitoring, homeland security, and other urban canyon application.

In the literature, there have been several studies on the lift generation mechanisms of flapping wings. A comprehensive review of those studies can be found in Ref. 1. Shyy et al. ${ }^{2}$ gave a detailed summary on challenges and issues associated with the MAV aerodynamics. Different aerodynamic formulations have been developed and applied for the studies of MAVs in hover or forward flight. One example of these developments is the unsteady aerodynamic formulation for insect-like flapping wings developed by Ansari el al. ${ }^{3,4}$, which was based on the nonlinear wake-integral equations that considered the effects of vortex shedding from both the airfoil's leading- and trailing-edges on lift generation. Gogulapati et al..$^{5}$ implemented this formulation and coupled with MSC.Marc for the study of the aeroelastic responses of a flapping wing. They also used this coupled formulation for analyzing flexible hawkmoth wings in hover ${ }^{6}$. On the experimental side, Dickinson and his co-workers conducted lowReynolds tests to measure the aerodynamic loads on a rigid airfoil ${ }^{7,8}$. The measured lift and drag data were fitted into empirical functions, which have been used in different studies (e.g., Refs. 9-11). The quasi-steady aerodynamics, while demonstrating some deviation from unsteady aerodynamic formulations ${ }^{7}$, is effective in dynamic and control studies of flapping MAVs.

Recently, some studies (e.g., Refs. 12-14) were performed to study the mechanisms of lift and thrust generation in the presence of wing flexibility. These numerical works were focused on the interactions between the fluids and the flapping elastic wings. The framework originally involved a finite-volume Navier-Stokes fluid dynamics solver and a finite-element structural dynamic solver based on geometrically nonlinear composite beam deformations. Different CFD/CSD coupling schemes were also included in the framework. In Ref. 13, the structural dynamic solver was updated with the application of a co-rotational shell finite-element, suitable for simulating shell-like flapping wings. With the introduced computational framework, Aono et al. ${ }^{15}$ studied the impact of spanwise flexibility on flapping wing aerodynamics for a rectangular wing with pure plunging motions. With the comparison to the experimental work performed by Heathcote et al. ${ }^{16}$, they were able to obtain good agreement between the numerical results and the experimental data in terms of instantaneous vertical tip displacement and thrust force on

\footnotetext{
${ }^{1}$ Postdoctoral Research Fellow (suw@umich.edu), Department of Aerospace Engineering, Senior Member AIAA.

${ }^{2}$ Professor (cesnik@umich.edu), Department of Aerospace Engineering, Associate Fellow AIAA.
} 
the flexible wing. They also summarized some thrust generation mechanisms with the spanwise flexibility of the wing.

In the above studies, the high-fidelity formulations were used to model the flapping wing aeroelasticity, which could provide more details about the load distributions on the lift wings. However, to understand the complete dynamic response of free-flight flapping wing MAVs, the coupled aeroelastic and flight dynamic characteristics of these flapping wing vehicles have to be considered.

Cesnik and Brown ${ }^{17,18}$ introduced a strain-based approach for the nonlinear modeling and coupled aeroelastic and flight dynamic analysis of very flexible aircraft with fixed-wings. It involved a low-order formulation that was capable of assessing the importance of structural nonlinear effects in a computationally effective manner. In Ref. 18, HALE aircraft were modeled using a rigid fuselage and highly flexible high-aspect-ratio wings. The strain-based beam formulation was solved in the time domain using an explicit integration scheme to obtain the flight dynamic response. Cesnik and $\mathrm{Su}^{19,20}$ extended the above work by considering the flexibility of the fuselage and vertical tails. Stability and roll maneuverability (using the aileron deflections only) were compared for models with different flexibility levels. Shearer and Cesnik ${ }^{21,22}$ completed the nonlinear flight dynamic equations in the strain-based beam formulation and updated the integration scheme with an implicit, modified Newmark method, which could provide long term numerical integration stability, compared to the previous explicit method.

More recently, Su and Cesnik ${ }^{23,24}$ performed the fully nonlinear analysis to study the time-domain characteristics of a flapping wing MAV by extending the low-order nonlinear aeroelastic simulation described above. Wing flapping kinematics is described using a set of Euler angles. The finite-state inflow aerodynamics ${ }^{25,26}$ and the unsteady aerodynamic formulation developed in Refs. 3 and 4 were compared in the study of Ref. 24. The interactions between the wing flapping kinematics and the vehicle flight dynamic response were observed from the simulation with symmetric wing flapping motions. With the free flight condition, the rigid-body motion of the vehicle could affect the local angle of attack of the airfoils and increase the stall margin. On the other side, the periodic wing flapping brought periodic pitching motion to the rigid body. The interaction significantly altered the trajectory of the vehicle. Vehicle maneuver could be achieved through asymmetric wing flapping actuations ${ }^{23}$.

Flight dynamic stability characteristics of flapping wing MAVs is still an open problem. Gao et al. ${ }^{27}$ studied the longitudinal dynamic stability of a hovering hawkmoth-type flapping wing MAV. In their work, aerodynamic sensitivities were evaluated based on CFD calculations of the aerodynamic loads. Eigenvalue analysis was carried out with the linearized system equations. They reported that the flapping wing model in that study was inherent dynamically stable without active controls. In contrast, Sun and Xiong ${ }^{28}$ indicated a dynamically unstable mode using a similar solution technique, while with a different wing and body kinematics. Analysis of hovering flapping MAVs may take advantage of its time-periodic feature. Rosenfeld and Wereley ${ }^{29}$ studied the elastic stability of a flapping wing using a linear beam theory with aerodynamic effects considered by viscous damping. The system was solved by using assumed mode shapes and the elastic stability of the time-periodic system with flapping motion was analyzed using Floquet theory.

In Refs. 27 and 28, CFD calculations were used to obtain aerodynamic loads and their sensitivities. Even though they can capture the pressure distribution correctly and give load estimation on the wings more accurately, CFD solutions always require a large amount of computational resources, which is not efficient in long-time aeroelastic simulations. In addition, flapping wing simulations involve large wing motions due to wing flapping actuations and rigid-body motion of the vehicle (in forward flight), which would require continuous linearization of CFD runs. Moreover, these large motions require significant deformation into the CFD grid, another time-consuming component of the solutions. In this paper, the analysis is based on the low-order aeroelastic formulation. The empirical aerodynamics of Ref. 7 will be applied for simplicity. As a preliminary study, this paper focuses on the flight dynamic stability of a hovering flapping wing MAV. The coupled nonlinear aeroelastic and flight dynamic formulation used in Refs. 23 and 24 is linearized and Floquet theory is used to facilitate the stability analysis. Both the longitudinal and lateral stability characteristics of the flapping wing MAV are explored.

\section{Theoretical Formulation}

Due to the interaction between flight dynamics and aeroelastic responses, the formulation includes six rigid-body and multiple flexible degrees of freedom. The structural members are allowed fully coupled three-dimensional bending, twisting, and extensional deformations. A quasi-steady aerodynamic formulation is incorporated for loads on all lifting surfaces. All these formulations are implemented in the University of Michigan's Nonlinear Aeroelastic Simulation Toolbox (UM/NAST). An overview of the formulations implemented in UM/NAST is described below. 


\section{A. Fundamental Descriptions}

As shown in Fig. 1, a global frame $G$ is defined, which is fixed on an inertial frame. A body frame $B$ is built in the global frame to describe the vehicle position and orientation, with $B_{x}$ pointing to the right wing, $B_{y}$ pointing towards the nose, and $B_{z}$ completing the triad. The position and orientation $(b)$ and the time derivatives $(\dot{b}$ and $\ddot{b})$ of the $B$ frame are defined as

$$
b=\left\{\begin{array}{c}
p_{B} \\
\theta_{B}
\end{array}\right\}, \quad \dot{b}=\beta=\left\{\begin{array}{c}
\dot{p}_{B} \\
\dot{\theta}_{B}
\end{array}\right\}=\left\{\begin{array}{c}
v_{B} \\
\omega_{B}
\end{array}\right\}, \quad \ddot{b}=\dot{\beta}=\left\{\begin{array}{c}
\ddot{p}_{B} \\
\ddot{\theta}_{B}
\end{array}\right\}=\left\{\begin{array}{c}
\dot{v}_{B} \\
\dot{\omega}_{B}
\end{array}\right\}
$$

where $p_{B}$ and $\theta_{B}$ are the body position and orientation, both resolved in the body frame $(B)$. Note that the origin of the body frame does not have to be the location of the vehicle's center of gravity.

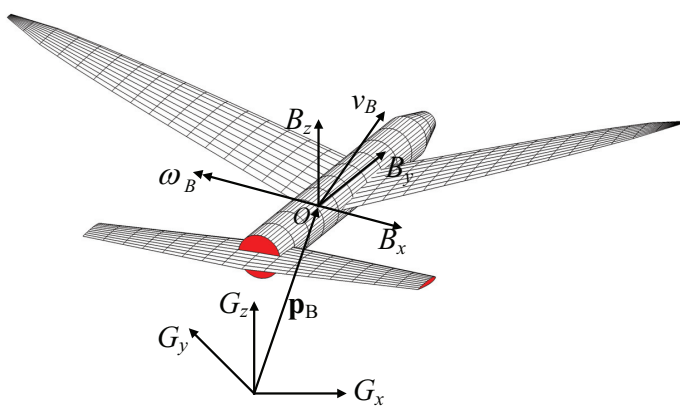

Figure 1. Global and body frames (for flight dynamics of the vehicle).

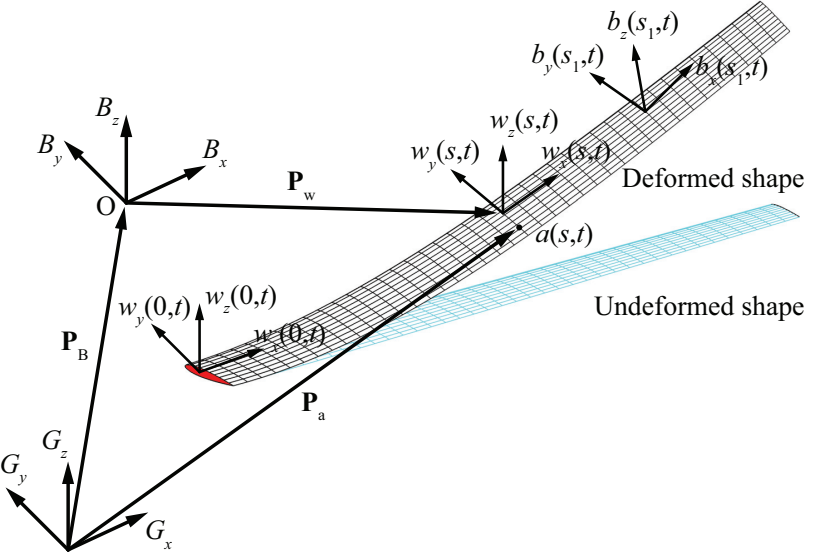

Figure 2. Flexible lifting surface frames within the body frame.

As described in Fig. 2, a local beam frame $w$ is built within the body frame, which is used to define the position and orientation of points along the beam reference line. $w_{x}, w_{y}$, and $w_{z}$ are base vectors of the beam frame, whose directions are pointing along the beam reference axis, toward the leading-edge, and normal to the beam surface, respectively, resolved in the body frame.

To model the elastic deformation of slender beams, a nonlinear beam element was developed ${ }^{18}$. The element has three nodes and four local strain degrees of freedom $\varepsilon$, which are extension, twist, and two bending curvatures of the beam reference line. Positions and orientations of each node along the beam are defined by a vector consisting of 12 components, which is denoted as

$$
h(s)^{T}=\left\{\left(p_{B}+p_{w}(s)\right)^{T}, \quad w_{x}(s)^{T}, \quad w_{y}(s)^{T}, \quad w_{z}(s)^{T}\right\}
$$

where, $p_{w}$ is the position of $w$ frame resolved in the $B$ frame. The derivative and variation of the dependent variable $h$ are related with those of the independent ones $(\varepsilon$ and $b)$ :

$$
\begin{aligned}
\delta h=J_{h \varepsilon} \delta \varepsilon+J_{h b} \delta b & \dot{h}=J_{h \varepsilon} \dot{\varepsilon}+J_{h b} \dot{b}=J_{h \varepsilon} \dot{\varepsilon}+J_{h b} \beta \\
d h=J_{h \varepsilon} d \varepsilon+J_{h b} d b & \ddot{h}=J_{h \varepsilon} \ddot{\varepsilon}+\dot{J}_{h \varepsilon} \dot{\varepsilon}+J_{h b} \dot{\beta}+\dot{J}_{h b} \beta
\end{aligned}
$$

where

$$
J_{h \varepsilon} \equiv \frac{\partial h}{\partial \varepsilon} \quad J_{h b} \equiv \frac{\partial h}{\partial b}
$$

which are Jacobians obtained from the beam kinematics ${ }^{17,19,22}$. 


\section{B. Modeling of Wing Flapping Kinematics}

To model the wing flapping kinematics (see Fig. 3), the relative rigid-body flapping motion between the base (non-flapping) member and the attached rotating (flapping) member can be described using Euler angles. The rotation matrix $R_{\varphi}$ between flapping and non-flapping members is obtained from the direction cosines between the two members

$$
R_{\varphi}=\left[\begin{array}{ccc}
\cos \Theta & -\sin \Theta & 0 \\
\sin \Theta & \cos \Theta & 0 \\
0 & 0 & 1
\end{array}\right]\left[\begin{array}{ccc}
\cos \Psi & 0 & \sin \Psi \\
0 & 1 & 0 \\
-\sin \Psi & 0 & \cos \Psi
\end{array}\right]\left[\begin{array}{ccc}
1 & 0 & 0 \\
0 & \cos \Phi & -\sin \Phi \\
0 & \sin \Phi & \cos \Phi
\end{array}\right]
$$

where $\Theta, \Phi$, and $\Psi$ are the yaw (sweeping), pitching, and roll (flapping) angles describing the rotations.

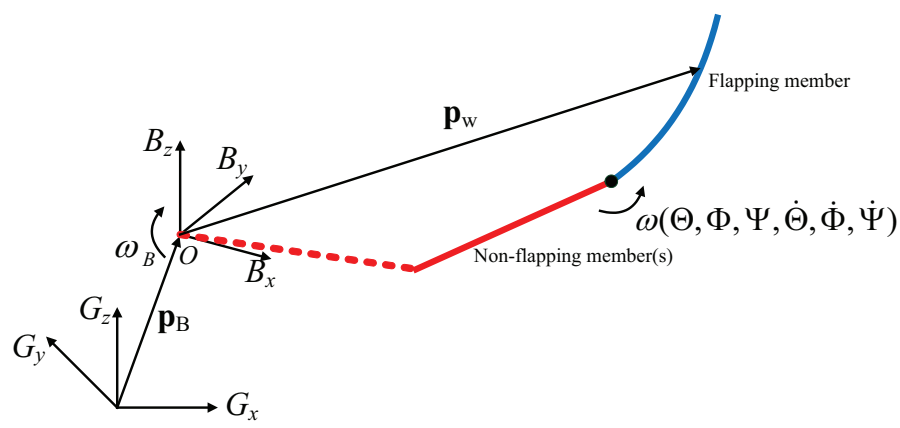

Figure 3. Kinematics relation between flapping and non-flapping members.

The rotational angular velocities and accelerations can be obtained from the above rotation matrix. When these Euler angles are small, the angular velocity about the local axis is approximately equal to the Euler angle rates. However, this relation does not hold for large rotation angles. In the current work, the angular velocity and accelerations are obtained through the following relations ${ }^{30}$

$$
\begin{gathered}
\boldsymbol{\omega}=\left\{\begin{array}{l}
\omega_{x} \\
\omega_{y} \\
\omega_{z}
\end{array}\right\}=\left\{\begin{array}{c}
\dot{\Phi} \cos \Psi \cos \Theta-\dot{\Psi} \sin \Theta \\
\dot{\Phi} \cos \Psi \sin \Theta+\dot{\Psi} \cos \Theta \\
\dot{\Theta}+\dot{\Phi} \sin \Psi
\end{array}\right\}=\left[\begin{array}{ccc}
0 & \cos \Psi \cos \Theta & -\sin \Theta \\
0 & \cos \Psi \sin \Theta & \cos \Theta \\
1 & \sin \Psi & 0
\end{array}\right]\left[\begin{array}{c}
\dot{\Theta} \\
\dot{\Phi} \\
\dot{\Psi}
\end{array}\right\} \\
\dot{\boldsymbol{\omega}}=\left\{\begin{array}{l}
\dot{\omega}_{x} \\
\dot{\omega}_{y} \\
\dot{\omega}_{z}
\end{array}\right\}=\left\{\begin{array}{c}
\ddot{\Phi} \cos \Psi \cos \Theta-\dot{\Phi} \dot{\Psi} \sin \Psi \cos \Theta-\dot{\Theta} \dot{\Phi} \cos \Psi \sin \Theta-\ddot{\Psi} \sin \Theta-\dot{\Theta} \dot{\Psi} \cos \Theta \\
\ddot{\Phi} \operatorname{sos} \Theta-\dot{\Phi} \dot{\Psi} \sin \Psi \sin \Theta+\dot{\Theta} \dot{\Phi} \cos \Psi \cos \Theta+\ddot{\Psi} \cos \Theta-\dot{\Theta} \dot{\Psi} \sin \Theta \\
\ddot{\Theta}+\ddot{\Phi} \sin \Psi+\dot{\Phi} \dot{\cos \Psi}
\end{array}\right\}
\end{gathered}
$$

\section{Equations of Motions}

The equations of motion of the system are obtained by applying the principle of virtual work. Following the same process described in Ref. 31, the elastic system equations of motion are derived as

$$
\left[\begin{array}{ll}
M_{F F} & M_{F B} \\
M_{B F} & M_{B B}
\end{array}\right]\left\{\begin{array}{l}
\ddot{\varepsilon} \\
\dot{\beta}
\end{array}\right\}+\left[\begin{array}{ll}
C_{F F} & C_{F B} \\
C_{B F} & C_{B B}
\end{array}\right]\left\{\begin{array}{l}
\dot{\varepsilon} \\
\beta
\end{array}\right\}+\left[\begin{array}{cc}
K_{F F} & 0 \\
0 & 0
\end{array}\right]\left\{\begin{array}{l}
\varepsilon \\
b
\end{array}\right\}=\left\{\begin{array}{l}
R_{F} \\
R_{B}
\end{array}\right\}
$$

where the generalized inertia, damping, and stiffness matrices are 


$$
\begin{aligned}
M_{F F}(\varepsilon) & =J_{h \varepsilon}^{T} M J_{h \varepsilon} & M_{F B}(\varepsilon) & =J_{h \varepsilon}^{T} M J_{h b} \\
M_{B F}(\varepsilon) & =J_{h b}^{T} M J_{h \varepsilon} & M_{B B}(\varepsilon) & =J_{h b}^{T} M J_{h b} \\
C_{F F}(\varepsilon, \dot{\varepsilon}, \beta) & =C+J_{h \varepsilon}^{T} M \dot{J}_{h \varepsilon} & C_{F B}(\varepsilon, \dot{\varepsilon}, \beta) & =J_{h \varepsilon}^{T} M H_{h b}+2 J_{h \varepsilon}^{T} M \dot{J}_{h b} \\
C_{B F}(\varepsilon, \dot{\varepsilon}, \beta) & =J_{h b}^{T} M \dot{J}_{h \varepsilon} & C_{B B}(\varepsilon, \dot{\varepsilon}, \beta) & =J_{h b}^{T} M H_{h b}+2 J_{h b}^{T} M \dot{J}_{h b} \\
K_{F F} & =K & &
\end{aligned}
$$

and the force vector is

$$
\begin{aligned}
\left\{\begin{array}{l}
R_{F} \\
R_{B}
\end{array}\right\} & \left\{\begin{array}{c}
K_{F F} \varepsilon^{0} \\
0
\end{array}\right\}+\left[\begin{array}{c}
J_{h s}^{T} \\
J_{h b}^{T}
\end{array}\right] N g+\left[\begin{array}{c}
J_{p \varepsilon}^{T} \\
J_{p b}^{T}
\end{array}\right] B^{F} F^{d i s t}+\left[\begin{array}{c}
J_{\theta \varepsilon}^{T} \\
J_{\theta b}^{T}
\end{array}\right] B^{M} M^{d i s t}+\left[\begin{array}{c}
J_{p \varepsilon}^{T} \\
J_{p b}^{T}
\end{array}\right] F^{p t}+\left[\begin{array}{c}
J_{\theta \varepsilon}^{T} \\
J_{\theta b}^{T}
\end{array}\right] M^{p t} \\
& +\left[\begin{array}{c}
J_{h \varepsilon}^{T} \\
J_{h b}^{T}
\end{array}\right] F^{a c c}+\left[\begin{array}{c}
J_{h \varepsilon}^{T} \\
J_{h b}^{T}
\end{array}\right] F^{c e n}+\left[\begin{array}{c}
J_{h \varepsilon}^{T} \\
J_{h b}^{T}
\end{array}\right] F^{c o r}+\left[\begin{array}{c}
J_{h s}^{T} \\
J_{h b}^{T}
\end{array}\right] F^{\text {int }}
\end{aligned}
$$

where $N, B^{F}$, and $B^{M}$ are the influence matrices for gravity force, distributed force, and distributed moments, respectively, coming from the numerical integration. The generalized force vector involves the effects from initial strains $\varepsilon^{0}$, gravity field $g$, distributed forces $F^{d i s t}$, distributed moments $M^{\text {dist }}$, point forces $F^{p t}$, and point moments $M^{p t}$. Additional inertia loads are applied to the flapping members, which include the contributions from the rotational acceleration $F^{a c c}$, centrifugal force $F^{c e n}$, Coriolis effects $F^{c o r}$, and interactions between the rotation of the body frame and the flapping members $F^{\text {int }}$, respectively. The details about these inertia loads can be found in Ref. 23 . The aerodynamic forces and moments are considered as distributed loads.

Two additional equations, describing the position and orientation of the body reference frame, are augmented to Eq. (7). Thus the body reference propagation can be conveniently solved. The coupled nonlinear aeroelastic and flight dynamic system equations of motion are then represented as Eq. $(10)^{22}$.

$$
\begin{gathered}
{\left[\begin{array}{cc}
M_{F F} & M_{F B} \\
M_{B F} & M_{B B}
\end{array}\right]\left\{\begin{array}{l}
\ddot{\varepsilon} \\
\dot{\beta}
\end{array}\right\}+\left[\begin{array}{ll}
C_{F F} & C_{F B} \\
C_{B F} & C_{B B}
\end{array}\right]\left\{\begin{array}{l}
\dot{\varepsilon} \\
\beta
\end{array}\right\}+\left[\begin{array}{cc}
K_{F F} & 0 \\
0 & 0
\end{array}\right]\left\{\begin{array}{l}
\varepsilon \\
b
\end{array}\right\}=\left\{\begin{array}{l}
R_{F} \\
R_{B}
\end{array}\right\}} \\
\dot{\zeta}_{B}=-\frac{1}{2} \Omega_{\zeta_{B}}(\beta) \zeta_{B} \\
\dot{P}_{B}=\left[\begin{array}{ll}
C^{G B}\left(\zeta_{B}\right) & 0
\end{array}\right] \beta
\end{gathered}
$$

where $\zeta_{B}$ is the quaternions describing the orientation of the body frame $B, P_{B}$ is the inertial position of the $B$ frame, and $C^{G B}$ is the rotation matrix from the body frame to global frame $G$.

\section{Empirical Aerodynamics}

The distributed loads, $F^{\text {dist }}$ and $M^{\text {dist }}$ in Eq. (9), are divided into aerodynamic loads and user supplied loads. The aerodynamic loads used in the current study are based on empirical expressions for lift and drag coefficients, which were obtained from Dickinson's low-Reynolds number experiments. ${ }^{7}$. The lift and drag applied on a thin 2-D flat plate section about the mid-chord are given by

$$
\begin{aligned}
l_{m c} & =\rho b V^{2} c_{l} \\
d_{m c} & =-\rho b V^{2} c_{d}
\end{aligned}
$$

where $b$ is the semichord of the airfoil, $V$ is the incoming flow velocity, with the consideration of airfoil motion due to the wing flapping actuation. The airfoil operates at a low Reynolds number regime with siginificant flow separation. The location of center of pressure is pushed backwords to the mid-chord location. The lift and drag coefficients are determined by the flowing empirical functions 


$$
\begin{aligned}
& c_{l}\left(\alpha_{e f f}\right)=0.225+1.58 \sin \left(2.13 \alpha_{e f f}-7.2\right) \\
& c_{d}\left(\alpha_{e f f}\right)=1.92-1.55 \cos \left(2.04 \alpha_{e f f}-9.82\right)
\end{aligned}
$$

The effective angle of attack of the airfoil, measured in degrees, is determined by the local velocities as

$$
\alpha_{e f f}=\frac{180}{\pi} \tan ^{-1}\left[-\frac{\dot{z}}{\dot{y}}+\left(\frac{1}{2} b-d\right) \frac{\dot{\alpha}}{\dot{y}}\right]
$$

where $d$ is the distance of the mid-chord in front of the reference axis, $-\dot{z} / \dot{y}$ (see Fig. 4) consists of the contribution from both the pitching angle and the unsteady plunging motion of the airfoil, and $\dot{\alpha}$ is the pitching rate of the airfoil.

Eventually, the lift and drag at the mid-chord need to be transferred to the wing reference axis and rotated to the body reference frame ${ }^{31}$ for solving the equations of motion. Note that finite span corrections are also included in the force distribution and may come, for instance, from a CFD solution of the problem.

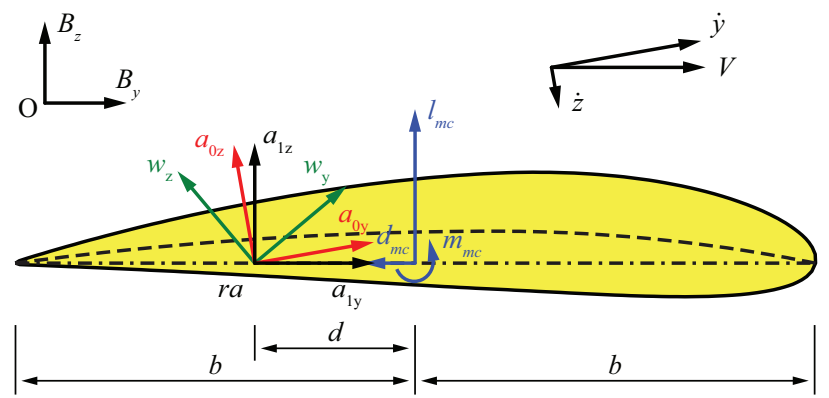

Figure 4. Airfoil coordinate system and velocity components.

\section{E. Linearization of the Nonlinear System Equations of Motion with Wing Flapping Motions}

The coupled nonlinear aeroelastic and flight dynamic system equations of motion are given by Eq. (10). Linearization of these equations is performed about the nonlinear equilibrium at a given time:

$$
\chi_{0}^{T}=\left\{\ddot{\varepsilon}_{0}^{T}, \dot{\varepsilon}_{0}^{T}, \varepsilon_{0}^{T}, \dot{\beta}_{0}^{T}, \beta_{0}^{T}, \zeta_{0}^{T}, P_{B 0}^{T}\right\}
$$

The nonlinear matrices in Eq. (10) can be perturbed about the equilibrium point. Note that the strain dependence of inertia and damping matrices is neglected, since its related to higer-order derivatives of the strain, which has less contribution to the whole linearized system. With the higher-order terms neglected, the nonlinear damping matrix is expanded as

$$
\begin{aligned}
& C_{F F}(\dot{\varepsilon}, \varepsilon, \beta)=C_{F F}\left(\dot{\varepsilon}_{0}, \varepsilon_{0}, \beta_{0}\right)+\left.\frac{\partial C_{F F}}{\partial \dot{\varepsilon}}\right|_{\dot{\varepsilon}_{0}} d \dot{\varepsilon}+\left.\frac{\partial C_{F F}}{\partial \beta}\right|_{\beta_{0}} d \beta \\
& C_{F B}(\dot{\varepsilon}, \varepsilon, \beta)=C_{F B}\left(\dot{\varepsilon}_{0}, \varepsilon_{0}, \beta_{0}\right)+\left.\frac{\partial C_{F B}}{\partial \dot{\varepsilon}}\right|_{\dot{\varepsilon}_{0}} d \dot{\varepsilon}+\left.\frac{\partial C_{F B}}{\partial \beta}\right|_{\beta_{0}} d \beta \\
& C_{B F}(\dot{\varepsilon}, \varepsilon, \beta)=C_{B F}\left(\dot{\varepsilon}_{0}, \varepsilon_{0}, \beta_{0}\right)+\left.\frac{\partial C_{B F}}{\partial \dot{\varepsilon}}\right|_{\dot{\varepsilon}_{0}} d \dot{\varepsilon}+\left.\frac{\partial C_{B F}}{\partial \beta}\right|_{\beta_{0}} d \beta \\
& C_{B B}(\dot{\varepsilon}, \varepsilon, \beta)=C_{B B}\left(\dot{\varepsilon}_{0}, \varepsilon_{0}, \beta_{0}\right)+\left.\frac{\partial C_{B B}}{\partial \dot{\varepsilon}}\right|_{\dot{\varepsilon}_{0}} d \dot{\varepsilon}+\left.\frac{\partial C_{B B}}{\partial \beta}\right|_{\beta_{0}} d \beta
\end{aligned}
$$

The load vector in Eq. (10) may include contributions from aerodynamic loads, gravity loads, and inertia loads due to wing flapping, given as: 


$$
\left\{\begin{array}{l}
R_{F} \\
R_{B}
\end{array}\right\}=\left\{\begin{array}{l}
R_{F}^{\text {aero }}(\ddot{\varepsilon}, \dot{\varepsilon}, \varepsilon, \dot{\beta}, \beta) \\
R_{B}^{\text {aero }}(\ddot{\varepsilon}, \dot{\varepsilon}, \varepsilon, \dot{\beta}, \beta)
\end{array}\right\}+\left\{\begin{array}{l}
R_{F}^{\text {grav }}(\zeta) \\
R_{B}^{\text {grav }}(\zeta)
\end{array}\right\}+\left\{\begin{array}{l}
R_{F}^{\text {acc }}(\varepsilon, \dot{\omega}) \\
R_{B}^{\text {acc }}(\varepsilon, \dot{\omega})
\end{array}\right\}+\left\{\begin{array}{l}
R_{F}^{c e n}(\varepsilon, \omega) \\
R_{B}^{c e n}(\varepsilon, \omega)
\end{array}\right\}+\left\{\begin{array}{l}
R_{F}^{\text {cor }}(\dot{\varepsilon}, \omega) \\
R_{B}^{\text {cor }}(\dot{\varepsilon}, \omega)
\end{array}\right\}+\left\{\begin{array}{l}
R_{F}^{\text {int }}(\varepsilon, \beta, \omega) \\
R_{B}^{\text {int }}(\varepsilon, \beta, \omega)
\end{array}\right\}
$$

which can also be expanded about the given state $\chi_{0}$ :

$$
\begin{aligned}
& R_{F}^{\text {aero }}=R_{F}^{\text {aero }}\left(\chi_{0}\right)+\left.\frac{\partial R_{F}^{\text {aero }}}{\partial \ddot{\varepsilon}}\right|_{\ddot{\varepsilon}_{0}} d \ddot{\varepsilon}+\left.\frac{\partial R_{F}^{\text {aero }}}{\partial \dot{\varepsilon}}\right|_{\dot{\varepsilon}_{0}} d \dot{\varepsilon}+\left.\frac{\partial R_{F}^{\text {aero }}}{\partial \varepsilon}\right|_{\varepsilon_{0}} d \varepsilon+\left.\frac{\partial R_{F}^{\text {aero }}}{\partial \dot{\beta}}\right|_{\dot{\beta}_{0}} d \dot{\beta}+\left.\frac{\partial R_{F}^{\text {aero }}}{\partial \beta}\right|_{\beta_{0}} d \beta \\
& R_{B}^{\text {aero }}=R_{B}^{\text {aero }}\left(\chi_{0}\right)+\left.\frac{\partial R_{B}^{\text {aero }}}{\partial \ddot{\varepsilon}}\right|_{\tilde{\varepsilon}_{0}} d \ddot{\varepsilon}+\left.\frac{\partial R_{B}^{\text {aero }}}{\partial \dot{\varepsilon}}\right|_{\dot{\varepsilon}_{0}} d \dot{\varepsilon}+\left.\frac{\partial R_{B}^{\text {aero }}}{\partial \varepsilon}\right|_{\varepsilon_{0}} d \varepsilon+\left.\frac{\partial R_{B}^{\text {aero }}}{\partial \dot{\beta}}\right|_{\dot{\beta}_{0}} d \dot{\beta}+\left.\frac{\partial R_{B}^{\text {aero }}}{\partial \beta}\right|_{\beta_{0}} d \beta \\
& R_{F}^{\text {grav }}=R_{F}^{\text {grav }}\left(\chi_{0}\right)+\left.\frac{\partial R_{F}^{\text {grav }}}{\partial \zeta}\right|_{\zeta_{0}} d \zeta \\
& R_{B}^{\text {grav }}=R_{B}^{\text {grav }}\left(\chi_{0}\right)+\left.\frac{\partial R_{B}^{\text {grav }}}{\partial \zeta}\right|_{\zeta_{0}} d \zeta \\
& R_{F}^{a c c}=R_{F}^{a c c}\left(\chi_{0}\right)+\left.\frac{\partial R_{F}^{a c c}}{\partial \varepsilon}\right|_{\varepsilon_{0}} d \varepsilon \\
& R_{B}^{a c c}=R_{B}^{a c c}\left(\chi_{0}\right)+\left.\frac{\partial R_{B}^{a c c}}{\partial \varepsilon}\right|_{\varepsilon_{0}} d \varepsilon \\
& R_{F}^{c e n}=R_{F}^{c e n}\left(\chi_{0}\right)+\left.\frac{\partial R_{F}^{c e n}}{\partial \varepsilon}\right|_{\varepsilon_{0}} d \varepsilon \\
& R_{B}^{c e n}=R_{B}^{c e n}\left(\chi_{0}\right)+\left.\frac{\partial R_{B}^{c e n}}{\partial \varepsilon}\right|_{\varepsilon_{0}} d \varepsilon \\
& R_{F}^{c o r}=R_{F}^{c o r}\left(\chi_{0}\right)+\left.\frac{\partial R_{F}^{c o r}}{\partial \dot{\varepsilon}}\right|_{\dot{\varepsilon}_{0}} d \dot{\varepsilon} \\
& R_{B}^{c o r}=R_{B}^{c o r}\left(\chi_{0}\right)+\left.\frac{\partial R_{B}^{c o r}}{\partial \dot{\varepsilon}}\right|_{\dot{\varepsilon}_{0}} d \dot{\varepsilon} \\
& R_{F}^{i n t}=R_{F}^{i n t}\left(\chi_{0}\right)+\left.\frac{\partial R_{F}^{i n t}}{\partial \varepsilon}\right|_{\varepsilon_{0}} d \varepsilon+\left.\frac{\partial R_{F}^{i n t}}{\partial \beta}\right|_{\beta_{0}} d \beta \\
& R_{B}^{\text {int }}=R_{B}^{\text {int }}\left(\chi_{0}\right)+\left.\frac{\partial R_{B}^{\text {int }}}{\partial \varepsilon}\right|_{\varepsilon_{0}} d \varepsilon+\left.\frac{\partial R_{B}^{\text {int }}}{\partial \beta}\right|_{\beta_{0}} d \beta
\end{aligned}
$$

With these expansions, the linearized equations are obtained by following the linearization process introduced in Ref. 31. 


$$
\begin{aligned}
& \left(\left.M_{F F}\right|_{\chi_{0}}-\left.\frac{\partial R_{F}^{\text {aero }}}{\partial \ddot{\varepsilon}}\right|_{\ddot{\varepsilon}_{0}}\right) \ddot{\varepsilon}+\left(\left.M_{F B}\right|_{\chi_{0}}-\left.\frac{\partial R_{F}^{\text {aero }}}{\partial \dot{\beta}}\right|_{\dot{\beta}_{0}}\right) \dot{\beta}+\left(\left.C_{F F}\right|_{\chi_{0}}+\left.\frac{\partial C_{F F}}{\partial \dot{\varepsilon}}\right|_{\dot{\varepsilon}_{0}} \dot{\varepsilon}_{0}+\left.\frac{\partial C_{F B}}{\partial \dot{\varepsilon}}\right|_{\dot{\varepsilon}_{0}} \beta_{0}-\left.\frac{\partial R_{F}^{\text {aero }}}{\partial \dot{\varepsilon}}\right|_{\dot{\varepsilon}_{0}}-\left.\frac{\partial R_{F}^{\text {cor }}}{\partial \dot{\varepsilon}}\right|_{\dot{\varepsilon}_{0}}\right) \dot{\varepsilon} \\
& +\left(\left.C_{F B}\right|_{\chi_{0}}+\left.\frac{\partial C_{F F}}{\partial \beta}\right|_{\beta_{0}} \dot{\varepsilon}_{0}+\left.\frac{\partial C_{F B}}{\partial \beta}\right|_{\beta_{0}} \beta_{0}-\left.\frac{\partial R_{F}^{\text {aero }}}{\partial \beta}\right|_{\beta_{0}}-\left.\frac{\partial R_{F}^{\text {int }}}{\partial \beta}\right|_{\beta_{0}}\right) \beta+\left(K_{F F}-\left.\frac{\partial R_{F}^{\text {aero }}}{\partial \varepsilon}\right|_{\varepsilon_{0}}-\left.\frac{\partial R_{F}^{\text {acc }}}{\partial \varepsilon}\right|_{\varepsilon_{0}}-\left.\frac{\partial R_{F}^{c e n}}{\partial \varepsilon}\right|_{\varepsilon_{0}}-\left.\frac{\partial R_{F}^{\text {int }}}{\partial \varepsilon}\right|_{\varepsilon_{0}}\right) \varepsilon \\
& =\left.\frac{\partial R_{F}^{\text {grav }}}{\partial \zeta}\right|_{\zeta_{0}} \zeta \\
& \left(\left.M_{B F}\right|_{\chi_{0}}-\left.\frac{\partial R_{B}^{\text {aero }}}{\partial \ddot{\varepsilon}}\right|_{\ddot{\varepsilon}_{0}}\right) \ddot{\varepsilon}+\left(\left.M_{B B}\right|_{\chi_{0}}-\left.\frac{\partial R_{B}^{\text {aero }}}{\partial \dot{\beta}}\right|_{\dot{\beta}_{0}}\right) \dot{\beta}+\left(\left.C_{B F}\right|_{\chi_{0}}+\left.\frac{\partial C_{B F}}{\partial \dot{\varepsilon}}\right|_{\dot{\varepsilon}_{0}} \dot{\varepsilon}_{0}+\left.\frac{\partial C_{B B}}{\partial \dot{\varepsilon}}\right|_{\dot{\varepsilon}_{0}} \beta_{0}-\left.\frac{\partial R_{B}^{\text {aero }}}{\partial \dot{\varepsilon}}\right|_{\dot{\varepsilon}_{0}}-\left.\frac{\partial R_{B}^{\text {cor }}}{\partial \dot{\varepsilon}}\right|_{\dot{\varepsilon}_{0}}\right) \dot{\varepsilon} \\
& +\left(\left.C_{B B}\right|_{\chi_{0}}+\left.\frac{\partial C_{B F}}{\partial \beta}\right|_{\beta_{0}} \dot{\varepsilon}_{0}+\left.\frac{\partial C_{B B}}{\partial \beta}\right|_{\beta_{0}} \beta_{0}-\left.\frac{\partial R_{B}^{\text {aero }}}{\partial \beta}\right|_{\beta_{0}}-\left.\frac{\partial R_{B}^{\text {int }}}{\partial \beta}\right|_{\beta_{0}}\right) \beta+\left(-\left.\frac{\partial R_{B}^{\text {aero }}}{\partial \varepsilon}\right|_{\varepsilon_{0}}-\left.\frac{\partial R_{B}^{\text {acc }}}{\partial \varepsilon}\right|_{\varepsilon_{0}}-\left.\frac{\partial R_{B}^{\text {cen }}}{\partial \varepsilon}\right|_{\varepsilon_{0}}-\left.\frac{\partial R_{B}^{\text {int }}}{\partial \varepsilon}\right|_{\varepsilon_{0}}\right) \varepsilon \\
& =\left.\frac{\partial R_{B}^{\text {grav }}}{\partial \zeta}\right|_{\zeta_{0}} \zeta
\end{aligned}
$$

The following definitions are introduced to simplify the above equations, i.e.,

$$
\begin{gathered}
\bar{M}_{F F}=\left.M_{F F}\right|_{\chi_{0}}-\left.\frac{\partial R_{F}^{\text {aero }}}{\partial \ddot{\varepsilon}}\right|_{\ddot{\varepsilon}_{0}}, \quad \bar{M}_{F B}=\left.M_{F B}\right|_{\chi_{0}}-\left.\frac{\partial R_{F}^{\text {aero }}}{\partial \dot{\beta}}\right|_{\dot{\beta}_{0}} \\
\bar{M}_{B F}=\left.M_{B F}\right|_{\chi_{0}}-\left.\frac{\partial R_{B}^{\text {aero }}}{\partial \ddot{\varepsilon}}\right|_{\ddot{\varepsilon}_{0}}, \quad \bar{M}_{B B}=\left.M_{B B}\right|_{\chi_{0}}-\left.\frac{\partial R_{B}^{\text {aero }}}{\partial \dot{\beta}}\right|_{\dot{\beta}_{0}} \\
\bar{C}_{F F}=\left.C_{F F}\right|_{\chi_{0}}+\left.\frac{\partial C_{F F}}{\partial \dot{\varepsilon}}\right|_{\dot{\varepsilon}_{0}} \dot{\varepsilon}_{0}+\left.\frac{\partial C_{F B}}{\partial \dot{\varepsilon}}\right|_{\dot{\varepsilon}_{0}} \beta_{0}-\left.\frac{\partial R_{F}^{\text {aero }}}{\partial \dot{\varepsilon}}\right|_{\dot{\varepsilon}_{0}}-\left.\frac{\partial R_{F}^{\text {cor }}}{\partial \dot{\varepsilon}}\right|_{\dot{\varepsilon}_{0}} \\
\bar{C}_{F B}=\left.C_{F B}\right|_{\chi_{0}}+\left.\frac{\partial C_{F F}}{\partial \beta}\right|_{\beta_{0}} \dot{\varepsilon}_{0}+\left.\frac{\partial C_{F B}}{\partial \beta}\right|_{\beta_{0}} \beta_{0}-\left.\frac{\partial R_{F}^{\text {eero }}}{\partial \beta}\right|_{\beta_{0}}-\left.\frac{\partial R_{F}^{\text {int }}}{\partial \beta}\right|_{\beta_{0}} \\
\bar{C}_{B F}=\left.C_{B F}\right|_{\chi_{0}}+\left.\frac{\partial C_{B F}}{\partial \dot{\varepsilon}}\right|_{\dot{\varepsilon}_{0}} \dot{\varepsilon}_{0}+\left.\frac{\partial C_{B B}}{\partial \dot{\varepsilon}}\right|_{\dot{\varepsilon}_{0}} \beta_{0}-\left.\frac{\partial R_{B}^{\text {aero }}}{\partial \dot{\varepsilon}}\right|_{\dot{\varepsilon}_{0}}-\left.\frac{\partial R_{B}^{\text {cor }}}{\partial \dot{\varepsilon}}\right|_{\dot{\varepsilon}_{0}} \\
\bar{C}_{B B}=\left.C_{B B}\right|_{\chi_{0}}+\left.\frac{\partial C_{B F}}{\partial \beta}\right|_{\beta_{0}} \dot{\varepsilon}_{0}+\left.\frac{\partial C_{B B}}{\partial \beta}\right|_{\beta_{0}} \beta_{0}-\left.\frac{\partial R_{B}^{\text {aero }}}{\partial \beta}\right|_{\beta_{0}}-\left.\frac{\partial R_{B}^{\text {int }}}{\partial \beta}\right|_{\beta_{0}}
\end{gathered}
$$




$$
\begin{gathered}
\bar{K}_{F F}=K_{F F}-\left.\frac{\partial R_{F}^{\text {aero }}}{\partial \varepsilon}\right|_{\varepsilon_{0}}-\left.\frac{\partial R_{F}^{a c c}}{\partial \varepsilon}\right|_{\varepsilon_{0}}-\left.\frac{\partial R_{F}^{\text {cen }}}{\partial \varepsilon}\right|_{\varepsilon_{0}}-\left.\frac{\partial R_{F}^{\text {int }}}{\partial \varepsilon}\right|_{\varepsilon_{0}} \\
\bar{K}_{B F}=-\left.\frac{\partial R_{B}^{\text {aero }}}{\partial \varepsilon}\right|_{\varepsilon_{0}}-\left.\frac{\partial R_{B}^{\text {acc }}}{\partial \varepsilon}\right|_{\varepsilon_{0}}-\left.\frac{\partial R_{B}^{c e n}}{\partial \varepsilon}\right|_{\varepsilon_{0}}-\left.\frac{\partial R_{B}^{\text {int }}}{\partial \varepsilon}\right|_{\varepsilon_{0}}
\end{gathered}
$$

From these, the linearized system equations can be written as

$$
\begin{array}{r}
\bar{M}_{F F} \ddot{\varepsilon}+\bar{M}_{F B} \dot{\beta}+\bar{C}_{F F} \dot{\varepsilon}+\bar{C}_{F B} \beta+\bar{K}_{F F} \varepsilon-\left.\frac{\partial R_{F}^{\text {grav }}}{\partial \zeta}\right|_{\zeta_{0}} \zeta=0 \\
\bar{M}_{B F} \ddot{\varepsilon}+\bar{M}_{B B} \dot{\beta}+\bar{C}_{B F} \dot{\varepsilon}+\bar{C}_{B B} \beta+\bar{K}_{B F} \varepsilon-\left.\frac{\partial R_{B}^{\text {grav }}}{\partial \zeta}\right|_{\zeta_{0}} \zeta=0 \\
\dot{\zeta}+\frac{1}{2} \Omega_{\zeta}\left(\beta_{0}\right) \zeta+\frac{1}{2}\left(\left.\frac{\partial \Omega_{\zeta}}{\partial \beta}\right|_{\beta_{0}} \beta\right) \zeta_{0}=0 \\
\dot{P}_{B}-\left[\begin{array}{ll}
C^{G B}\left(\zeta_{0}\right) & 0
\end{array}\right] \beta-\left[\left(\left.\frac{\partial C^{G B}}{\partial \zeta}\right|_{\zeta_{0}} ^{\zeta}\right) 0\right] \beta_{0}=0
\end{array}
$$

Finally, Eq. (30) can be put into state-space form

$$
\begin{aligned}
\left\{\begin{array}{ccccc}
\dot{\varepsilon}^{T} & \ddot{\varepsilon}^{T} & \dot{\beta}^{T} & \dot{\zeta}^{T} & \dot{P}_{B}^{T}
\end{array}\right\}^{T} & =Q_{1}^{-1}(t) Q_{2}(t)\left\{\begin{array}{lllll}
\varepsilon^{T} & \dot{\varepsilon}^{T} & \beta^{T} & \zeta^{T} & P_{B}^{T}
\end{array}\right\}^{T} \\
& =A(t)\left\{\begin{array}{lllll}
\varepsilon^{T} & \dot{\varepsilon}^{T} & \beta^{T} & \zeta^{T} & P_{B}^{T}
\end{array}\right\}^{T}
\end{aligned}
$$

or

$$
\dot{x}(t)=A(t) x(t)
$$

where

$$
\begin{gathered}
x(t)^{T}=\left\{\begin{array}{ccccc}
\varepsilon^{T} & \dot{\varepsilon}^{T} & \beta^{T} & \zeta^{T} & P_{B}^{T}
\end{array}\right\} \\
Q_{1}=\left[\begin{array}{ccccc}
I & 0 & 0 & 0 & 0 \\
0 & \bar{M}_{F F} & \bar{M}_{F B} & 0 & 0 \\
0 & \bar{M}_{B F} & \bar{M}_{B B} & 0 & 0 \\
0 & 0 & 0 & I & 0 \\
0 & 0 & 0 & 0 & I
\end{array}\right], Q_{2}=\left[\begin{array}{ccccc}
0 & I & 0 & 0 & 0 \\
-\bar{K}_{F F} & -\bar{C}_{F F} & -\bar{C}_{F B} & \left.\frac{\partial R_{F}^{\text {grav }}}{\partial \zeta}\right|_{\zeta_{0}} & 0 \\
-\bar{K}_{B F} & -\bar{C}_{B F} & -\bar{C}_{B B} & \left.\frac{\partial R_{B}^{\text {grav }}}{\partial \zeta}\right|_{\zeta_{0}} & 0 \\
0 & 0 & -\left.\frac{1}{2} \frac{\partial \Omega_{\zeta}}{\partial \beta}\right|_{\beta_{0}} & \frac{1}{2} \Omega_{\zeta}\left(\beta_{0}\right) & 0 \\
0 & 0 & {\left[\begin{array}{ccc}
C^{G B}\left(\zeta_{0}\right) & 0
\end{array}\right]\left[\begin{array}{ccc}
\left.\frac{\partial C^{G B}}{\partial \zeta}\right|_{\zeta_{0}} & 0
\end{array}\right] \beta_{0}} & 0
\end{array}\right]
\end{gathered}
$$




\section{F. Flight Dynamic Stability}

Consider the periodic feature of the hovering flapping wing MAV, the stability of the system can be evaluated by using the Floquet theory ${ }^{32}$. The $A$ matrix in Eq. (32) is periodic due to wing flapping motions. Defining the transition matrix between two time solutions as

$$
x(t)=\Phi_{A}\left(t, t_{0}\right) x\left(t_{0}\right)
$$

It natually satisfies the following relation:

$$
\dot{\Phi}_{A}\left(t, t_{0}\right)=A(t) \Phi_{A}\left(t, t_{0}\right)
$$

According to the Floquet theory, the stability of the system represented by Eq. (32) is determined by the eigenvalues of transition matrix of one period $\Phi_{A}\left(t_{0}+T, t_{0}\right)$. Let $\Lambda_{s}(s=1,2,3, \ldots, n)$ be the eigenvalues of $\Phi_{A}\left(t_{0}+T, t_{0}\right)$. The stability criterion for the system can be stated in terms of the magnitude of $\Lambda_{s}^{33}$, i.e.,

$$
\left|\Lambda_{s}\right|<1 \quad, \quad s=1,2,3, \ldots, n
$$

The damping and frequency of the corresponding eigenmodes can be obtained from $\Lambda_{s}$, given as

$$
\begin{aligned}
\xi_{s} & =\frac{1}{2 T} \ln \left[\left(\operatorname{Re}\left(\Lambda_{s}\right)\right)^{2}+\left(\operatorname{Im}\left(\Lambda_{s}\right)\right)^{2}\right] \\
\omega_{s} & =\frac{1}{T} \tan ^{-1}\left(\frac{\operatorname{Im}\left(\Lambda_{s}\right)}{\operatorname{Re}\left(\Lambda_{s}\right)}\right)
\end{aligned}
$$

To calculate $\Phi_{A}\left(t_{0}+T, t_{0}\right)$, the period is divided into $k$ equivalent time intervals $\Delta t$. Within each time interval, the system matrix $A$ is assumed constant. Therefore, the system can be treated as a linear time-invariant system as

$$
\dot{x}_{i}(t)=A_{i} x_{i}(t) \quad, \quad i=1, \ldots, k
$$

and its solution over one period is obtained as

$$
x\left(t_{0}+T\right)=e^{A_{k} \Delta t} \cdots e^{A_{2} \Delta t} e^{A_{1} \Delta t} x\left(t_{0}\right)
$$

The transition matrix over one period is then determined as

$$
\Phi_{A}\left(t_{0}+T, t_{0}\right)=e^{A_{k} \Delta t} \cdots e^{A_{2} \Delta t} e^{A_{1} \Delta t}=\prod_{i=1}^{k} e^{A_{i} \Delta t}
$$

In practice, a numerical simulation of one period can be performed, from which the system matrix $A$ of the linearized system at each time interval can be obtained. And the transition matrix of this period is obtained from Eq. (41).

\section{Numerical Study and Discussion}

With the theoretical formulation numerically implemented in UM/NAST, a hovering flapping wing MAV is numerically studied to exemplify the process and the capabilities of the method. The impact of wing rigidity and inertia on the flight dynamic stability of the flapping wing MAV is explored for this particular case. 


\section{A. Description of Baseline Flapping Wing MAV Model}

The baseline of the flapping wing MAV is shown in Fig. 5. The wing has an elliptic Zimmerman planform. Each of the wings has a span of $75 \mathrm{~mm}$ and a chord length of $25 \mathrm{~mm}$ at the root. The Zimmerman geometry is determined by two ellipses meeting at the quarter of chord using the following equations

$$
\begin{aligned}
& \frac{x^{2}}{L_{w}^{2}}+\frac{y^{2}}{\left(c_{r} / 4\right)^{2}}=1 \\
& \frac{x^{2}}{L_{w}^{2}}+\frac{y^{2}}{\left(3 c_{r} / 4\right)^{2}}=1
\end{aligned}
$$

where $L_{w}$ is the wing span and $c_{r}$ is the chord length at the wing root. The pivot point of the two wings is located at the root of the leading edge, and the beam reference axis is then defined along the wing leading edge. There is a distance of $12 \mathrm{~mm}$ between the two wings pivot points, between which the vehicle' rigid-body reference frame $B$ is defined. Note that the fuselage in the figure is for visualization purpose only. The inertia of the rigid-body fuselage is lumped at $2 \mathrm{~mm}$ aft of the $B$ frame, as indicated by the black point in the figure. Its properties are listed in Table 1. The distribution of wing properties are listed in the Apendix.

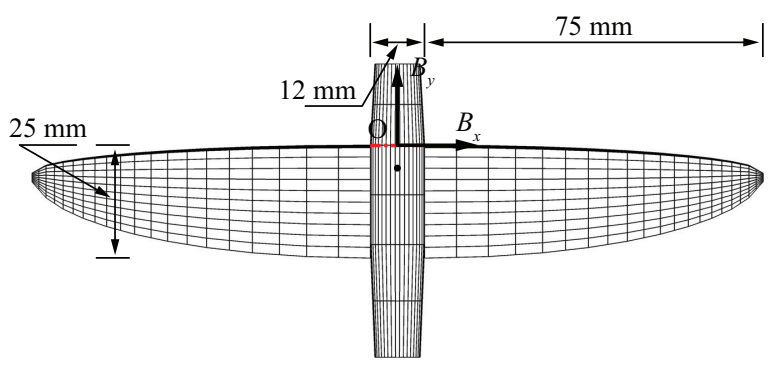

Figure 5. Geometry of the flapping wing MAV (with no flapping actuations).

Table 1. Rigid-body properties of the flapping wing vehicle

\begin{tabular}{lcc}
\hline \hline Property & Value & Units \\
\hline Rigid-body mass & $2.562 \times 10^{-4}$ & $\mathrm{~kg}$ \\
Mass moment of inertia about $B_{x}$ & $12.75 \times 10^{-5}$ & $\mathrm{~kg} . \mathrm{m}^{2}$ \\
Mass moment of inertia about $B_{y}$ & $5.000 \times 10^{-5}$ & $\mathrm{~kg} . \mathrm{m}^{2}$ \\
Mass moment of inertia about $B_{z}$ & $12.75 \times 10^{-5}$ & $\mathrm{~kg} \cdot \mathrm{m}^{2}$ \\
\hline \hline
\end{tabular}

\section{B. Description of Wing Flapping Kinematics}

The wing flapping actuation features 2-D sweeping and pitching motions at the wing root. The prescribed sweeping and pitching angles at the wing root are determined by

$$
\begin{aligned}
& \delta(t)=\delta_{0} \cos (2 \pi f t) \\
& \alpha(t)=90-\alpha_{0} \sin (2 \pi f t)
\end{aligned}
$$

where the constants in the sweeping and pitching equations are $\delta_{0}=30^{\circ}$ and $\alpha_{0}=45^{\circ}$, respectively. The flapping frequency $f$ is $10 \mathrm{~Hz}$. The kinematics is chosen such that the aircraft is able to maintain its hovering state with the wing flapping motion (see Fig. 6). 


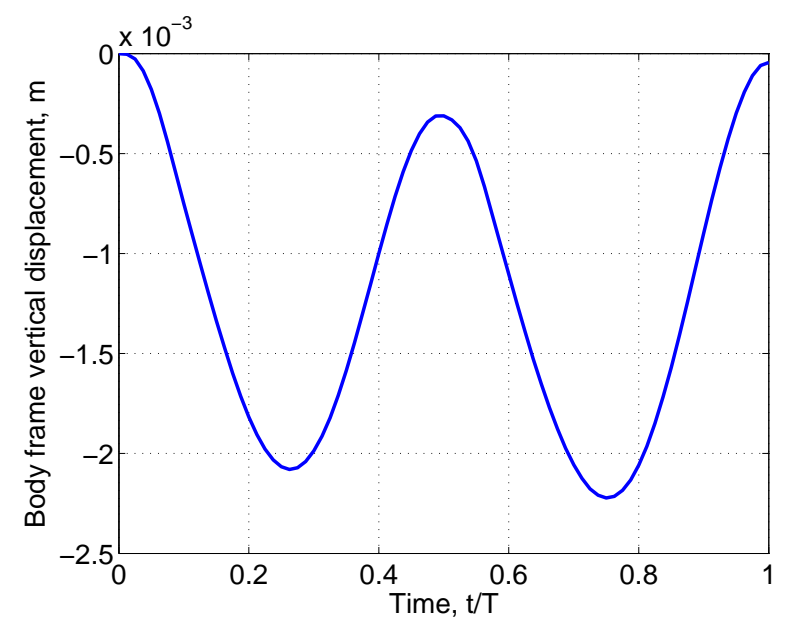

Figure 6. Vertical displacement of the body frame.

\section{Impact of Wing Rigidity on the Flight Stability}

The wing rigidity is varied in this study to assess its impact on the MAV's flight dynamic stability, while the wing inertia is kept the same as the baseline value. With different wing stiffness, the longitudinal and lateral stabilities of the flapping wing MAV are evaluated using the approach introduced in this paper. The eigenvalues of the system transition matrices are listed in Table 2. The damping and frequencies of the longitudinal and lateral modes are obtained through Eq. (38), and the corresponding root loci are plotted in Figs. 7 and 8.

Table 2. Eigenvalues of system transition matrices with different wing rigidities

\begin{tabular}{cccc}
\hline \hline & $0.1 \times$ rigidity & Baseline & $100 \times$ rigidity \\
\hline \multirow{3}{*}{ Longitudinal } & 1.713425 & 1.519570 & 1.766608 \\
& 0.279942 & 0.369550 & 0.187536 \\
& $0.998658 \pm 0.034946 i$ & $0.998481 \pm 0.018848 i$ & $0.993469 \pm 0.073961 i$ \\
\hline \multirow{3}{*}{ lateral } & 0.855871 & 0.769134 & 0.819769 \\
& 0.998623 & 0.997932 & 1.001533 \\
& $1.080328 \pm 0.150944 i$ & $1.020787 \pm 0.130288 i$ & $1.119045 \pm 0.227721 i$ \\
\hline \hline
\end{tabular}
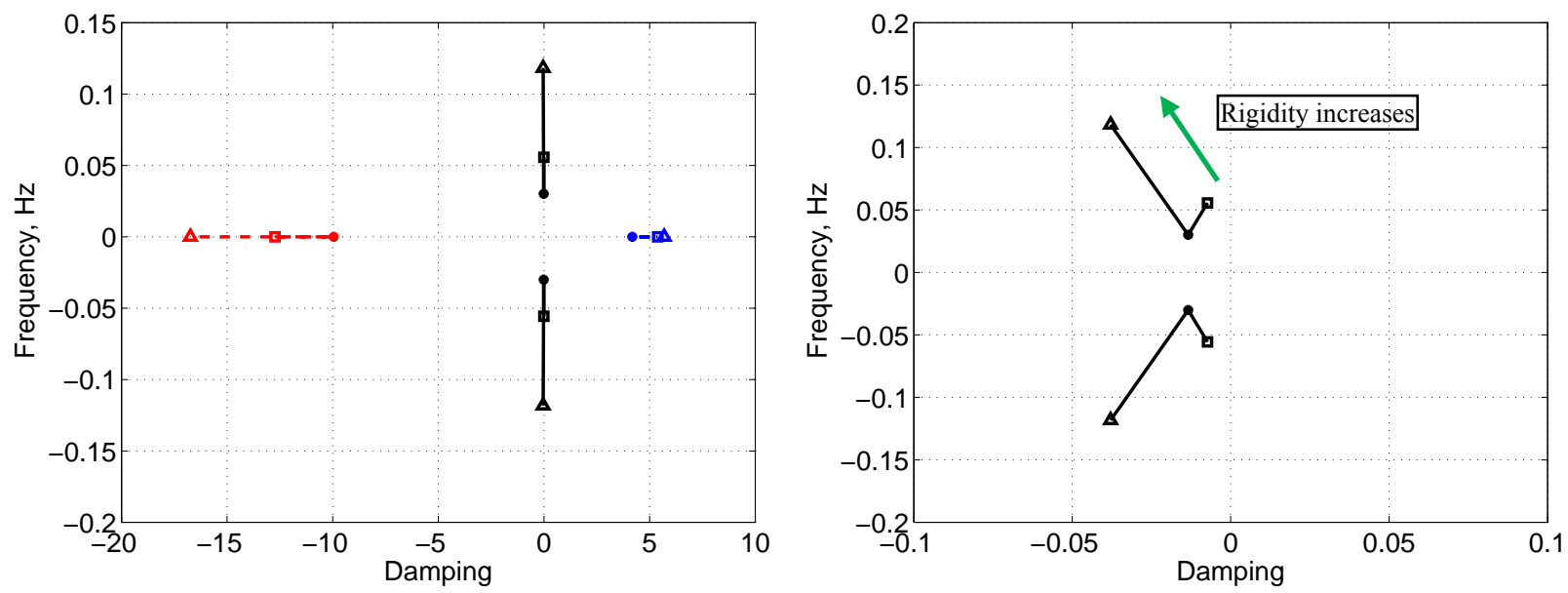

Figure 7. Root loci of the longitudinal modes with rigidity variation (square: $0.1 \times$ rigidity, star: Baseline, triangle: $100 \times$ rigidity, zoomed on the right hand side). 
From the plot of the root loci for the longitudinal modes (Fig. 7), one can clearly identify one oscillatory mode and two non-oscillatory modes. For fixed-wing wing-body-tail configurations, their longitudinal modes usually consist of a slightly-damped phugoid mode and a highly-damped short-period mode. For this flapping-wing MAV, the slightly-damped low-frequency phugoid mode still exists. The increase of wing stiffness tends to move the root locus of this mode away from the origin point, which indicates the increase of the stability margin for this mode. However, the short-period mode does not exist anymore. Instead, two non-oscillatory modes participate, one of which is unstable. As the time for the unstable mode to double its amplitude is relatively short (in the order of 0.14 s), indicating a quick growing divergent motion, a dedicated controller will be required to maintain its flight.

It is worth noticing here that in the studies of Gao et al. ${ }^{27}$ and Sun and Xiong ${ }^{28}$, similar modes were also found, while the stability characteristics of each individual mode were not the same as the ones in the current study. Note that the aircraft properties and wing flapping kinematics are different between these studies, which might contribute to the difference in the stability characteristics of these modes.

In the lateral direction (Fig. 8), one can identify a stable rolling mode, an unstable Dutch roll mode, and a spiral model, whose stability boundary is impacted by the change of wing stiffness. The time for the Dutch roll mode to double its amplitude is longer than the flapping cycle.
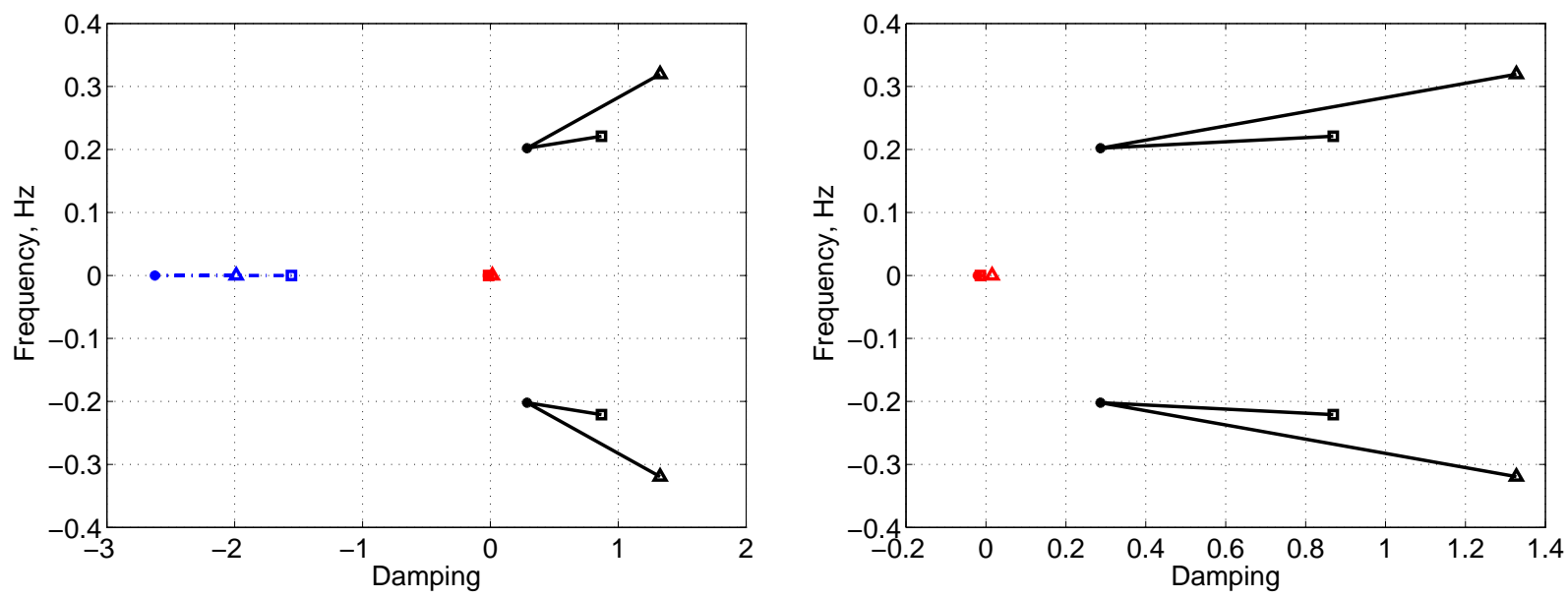

Figure 8. Root loci of the lateral modes with rigidity variation (square: $0.1 \times$ rigidity, star: Baseline, triangle: $100 \times$ rigidity, zoomed on the right hand side).

\section{Impact of Wing Inertia on the Flight Stability}

In this section, the wing inertia is varied to assess its impact on the MAV's flight dynamic stability, while the wing rigidity is kept unchanged from the baseline configuration. With different wing inertias, the longitudinal and lateral stabilities of the flapping wing MAV are evaluated. The eigenvalues of the system transition matrices are listed in Table 3. The damping and frequencies of the longitudinal and lateral modes are obtained through Eq. (38), and the corresponding root loci are plotted in Figs. 9 and 10.

Table 3. Eigenvalues of system transition matrices with different wing inertias

\begin{tabular}{cccc}
\hline \hline & $0.2 \times$ inertia & Baseline & $5 \times$ inertia \\
\hline \multirow{4}{*}{ Longitudinal } & $-1.887546 \times 10^{3}$ & 1.519570 & 1.518652 \\
& $-1.217075 \times 10^{-3}$ & 0.369550 & 0.445772 \\
& $0.999926 \pm 0.007313 i$ & $0.998481 \pm 0.018848 i$ & $0.997399 \pm 0.035413 i$ \\
\hline \multirow{3}{*}{ lateral } & $3.351351 \times 10^{-5}$ & 0.769134 & 0.860864 \\
& 0.992928 & 0.997932 & 1.000647 \\
& $0.997466 \pm 0.229441 i$ & $1.020787 \pm 0.130288 i$ & $1.073373 \pm 0.150684 i$ \\
\hline \hline
\end{tabular}

From the plot of the root loci for the longitudinal modes (Fig. 9) with different wing inertias, it is evident that the wing inertia variation has more impact on the two non-oscillatory modes, whose damping is significantly reduced with the increase of wing inertia. The oscillatory mode is always stable with the increase of wing inertia. From the plot of the lateral modes' root loci (Fig. 10), it can be seen the rolling mode is still stable, even though its root 
damping is reduced with the increase of wing inertia. The Dutch roll mode is always unstable, while the stability boundary of the spiral model is marginal.
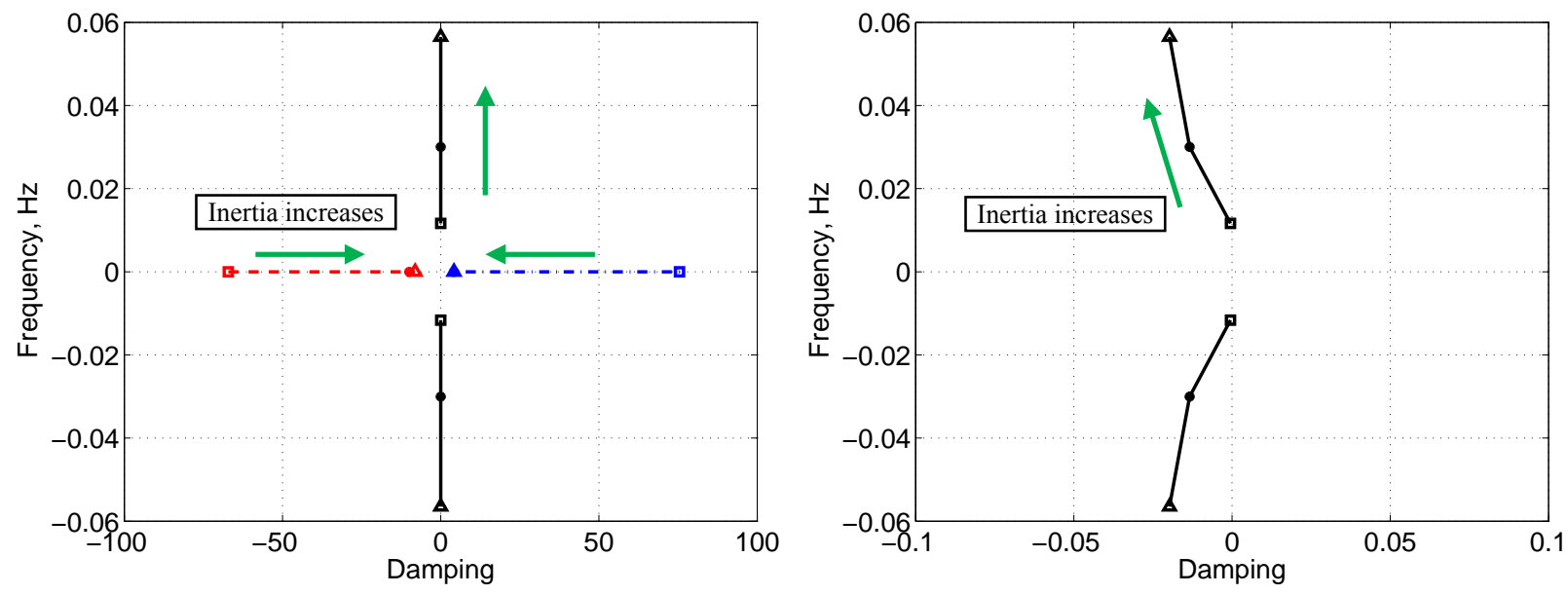

Figure 9. Root loci of the longitudinal modes with inertia variation (square: $0.2 \times$ inertia, star: Baseline, triangle: $5 \times$ inertia, zoomed on the right hand side).
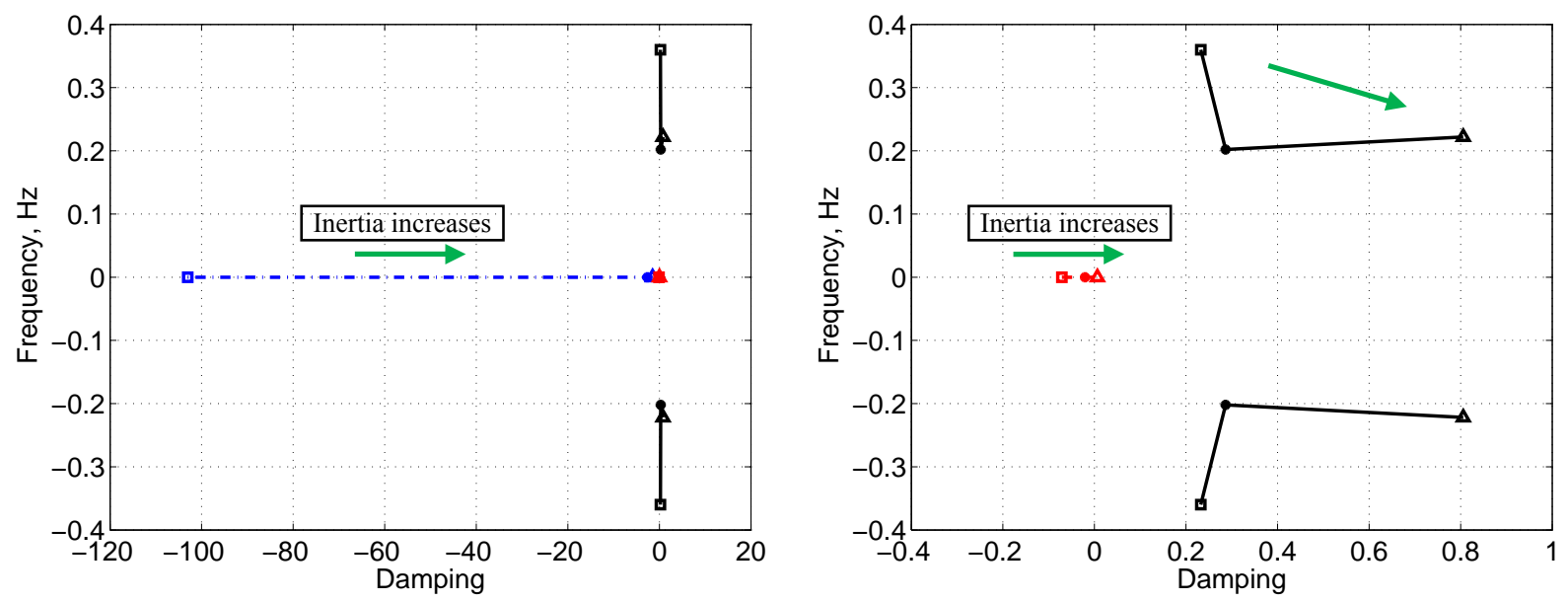

Figure 10. Root loci of the lateral modes with inertia variation (square: $0.2 \times$ inertia, star: Baseline, triangle: $5 \times$ inertia, zoomed on the right hand side).

\section{Concluding Remarks}

An approach to evaluate the flight dynamic stability of flapping wing Micro Air Vehicles (MAVs) was introduced in this paper. It was based on a low-order aeroelastic formulation. First, the nonlinear aeroelastic behavior of flapping wing MAVs was represented by a strain-based nonlinear beam formulation, coupled with empirical quasi-steady aerodynamic equations. Wing flapping kinematics was described by using a set of Euler angles. Nonlinear equations of motion for the body frame attached to the vehicle (not necessarily at its center of gravity) were used to complete the coupled aeroelastic and flight dynamic formulation. The nonlinear equations were linearized about each nonlinear equilibrium state within a flapping period. Since the system is time-periodic, the stability of a hovering flapping wing MAV was evaluated based on the transition matrix over a flapping period using the Floquet theory.

In the numerical studies on a flapping wing MAV with the Zimmerman planform, the traditional short-period longitudinal mode did not exist. Instead, there were two non-oscillatory longitudinal modes, one of which was found to be unstable and with large positive damping. The oscillatory mode, similar to the traditional phugoid mode, could 
be seen in the eigenmodes. Its stability margin was increased with the increase of wing stiffness and inertia. In the lateral direction, the Dutch roll mode was always unstable with the change of wing stiffness and inertia.

Above all, this paper discussed an effective method to analyze the flight dynamic stability of time-periodic flapping wing Micro Air Vehicles (MAVs) in hover. The solution is based on a low-order representation of the coupled aeroelastic and flight dynamic behavior of the flapping MAVs. The framework is flexible enough that it can accommodate new aerodynamic modules as they become available from CFD surrogate methods.

\section{Acknowledgments}

The work has been supported by the Air Force Office of Scientific Research's Multidisciplinary University Research Initiative (MURI) and by the Michigan/AFRL/Boeing Collaborative Center in Aeronautical Sciences. The program manager is Dr. Doug Smith.

\section{Appendix}

The wing rigidty and inertia properties are listed below:
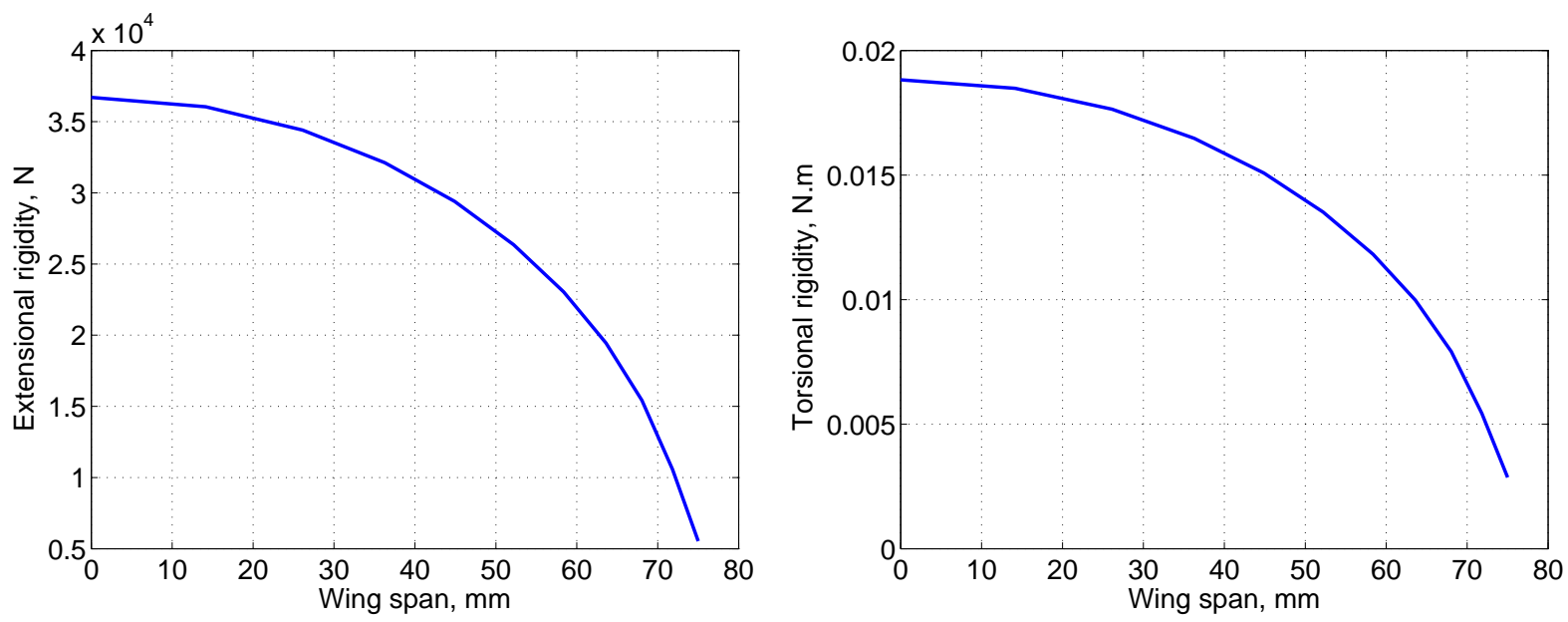

Figure A1. Distribution of wing extensional rigidity. Figure A2. Distribution of wing torsional rigidity.

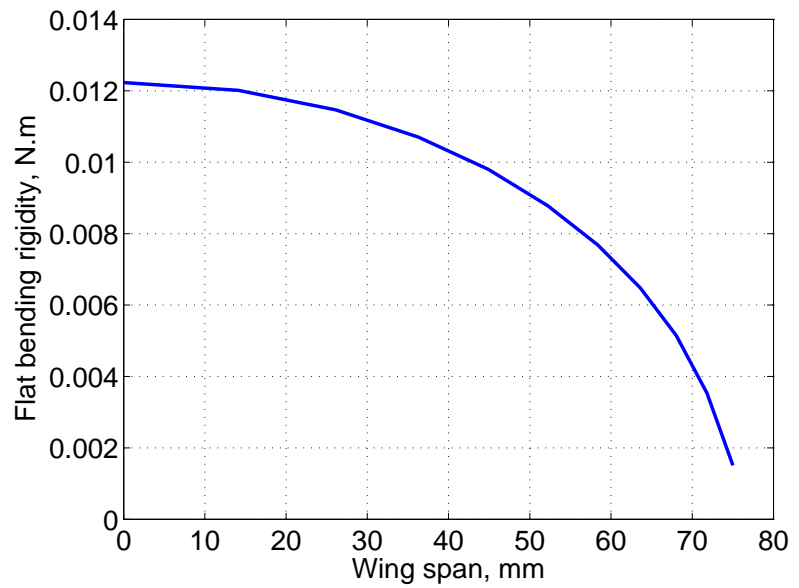

Figure A3. Distribution of wing flat bending rigidity.

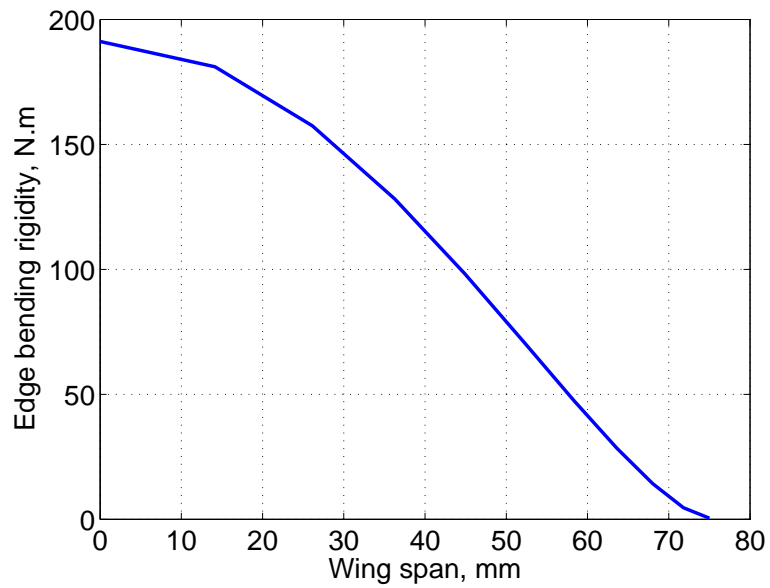

Figure A4. Distribution of wing edge bending rigidity. 


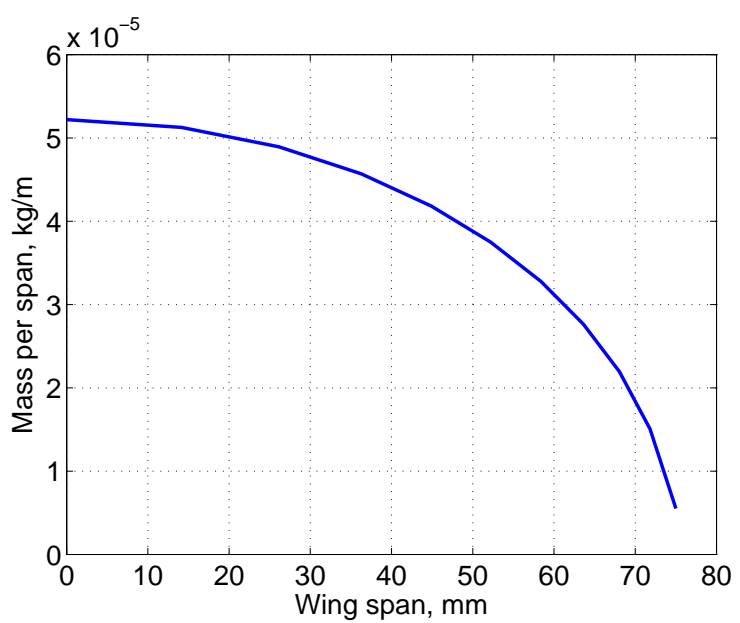

Figure A5. Distribution of wing mass.

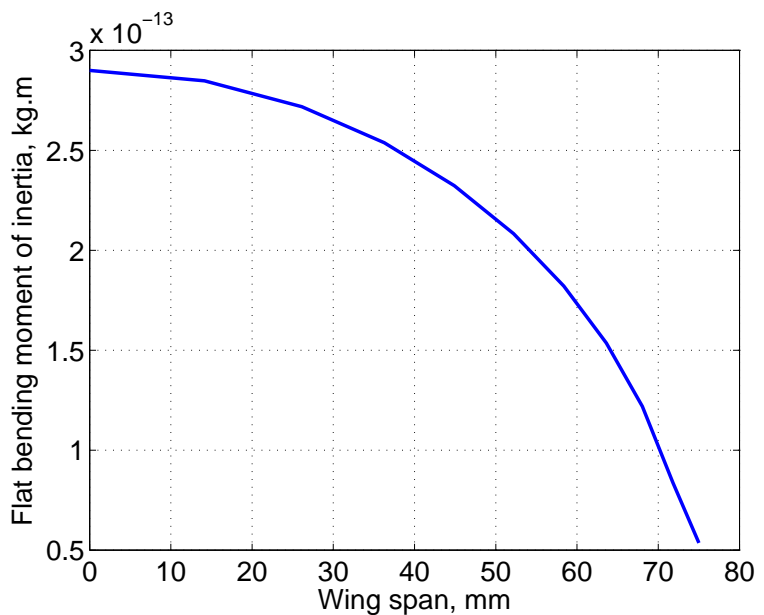

Figure A7. Distribution of wing flat bending moment of inertia.

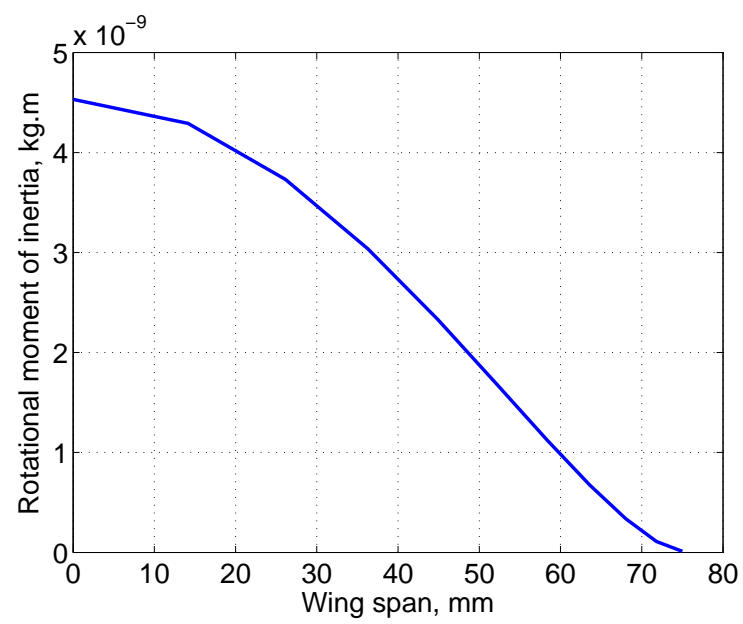

Figure A6. Distribution of wing rotational moment of inertia.

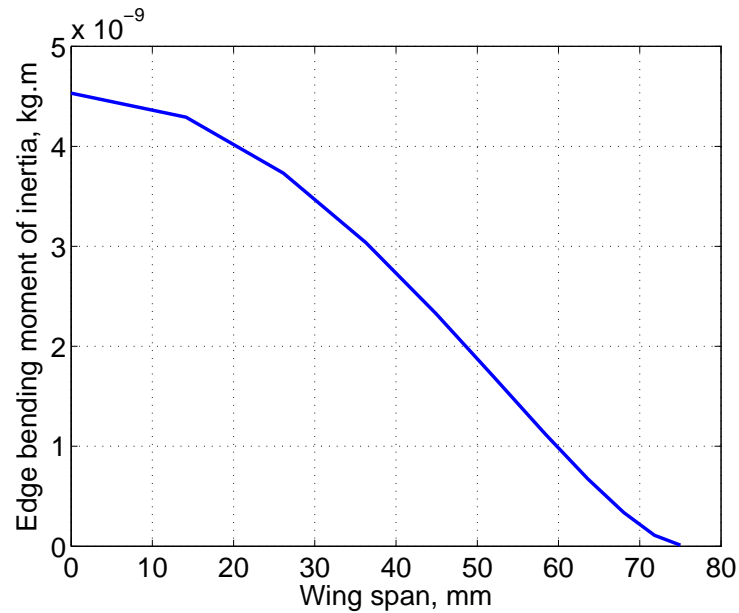

Figure A8. Distribution of wing edge bending moment of inertia.

\section{References}

${ }^{1}$ Shyy, W., Lian, Y., Tang, J., Viieru, D. and Liu, H., Aerodynamics of Low Reynolds Number Flyers, Cambridge University Press, New York, 2007.

${ }^{2}$ Shyy, W., Lian, Y., Tang, J., Liu, H., Trizila, P., Stanford, B., Bernal, L., Cesnik, C. E. S., Friedmann, P. P. and Ifju, P., "Computational Aerodynamics of Low Reynolds Number Plunging, Pitching and Flexible Wings for MAV Applications," AIAA-2008-0523, 46th AIAA Aerospace Sciences Meeting and Exhibit, Reno, NV, Jan. 7-10, 2008.

${ }^{3}$ Ansari, S. A., Żbikowski, R. and Knowles, K., "Non-linear Unsteady Aerodynamic Model for Insect-Like Flapping Wings in Hover. Part 1: Methodology and Analysis," Proceedings of the Institution of Mechanical Engineers, Part G: Journal of Aerospace Engineering, Vol. 220, No. 2, 2006, pp. 61-83.

${ }^{4}$ Ansari, S. A., Żbikowski, R. and Knowles, K., "Non-linear Unsteady Aerodynamic Model for Insect-Like Flapping Wings in Hover. Part 2: Implementation and Validation," Proceedings of the Institution of Mechanical Engineers, Part G: Journal of Aerospace Engineering, Vol. 220, No. 3, 2006, pp. 169-186.

${ }^{5}$ Gogulapati, A., Friedmann, P. P. and Shyy, W., "Nonlinear Aeroelastic Effects in Flapping Wing Micro Air Vehicles," AIAA-2008-1817, 49th AIAA/ASME/ASCE/AHS/ASC Structures, Structural Dynamics, and Materials Conference, Schaumburg, IL, Apr. 7-10, 2008.

${ }^{6}$ Gogulapati, A., Friedmann, P. P. and Shyy, W., “Approximate Aeroelastic Analysis of Flapping Wings in Hover,” IFASD2009-143, International Forum on Aeroelasticity and Structural Dynamics 2009, Seattle, WA, June 21-25, 2009.

${ }^{7}$ Dickinson, M. H., Lehmann, F.-O. and Sane, S. P., "Wing Rotation and the Aerodynamic Basis of Insect Flight," Science, Vol. 284, No. 5422, 1999, pp. 1954-1960. 
${ }^{8}$ Sane, S. P. and Dickinson, M. H., "The Aerodynamic Effects of Wing Rotation and a Revised Quasi-Steady Model of Flapping Flight," Journal of Experimental Biology, Vol. 205, No. 8, 2002, pp. 1087-1096.

${ }^{9}$ Doman, D. B., Oppenheimer, M. W. and Sigthorsson, D. O., "Wingbeat Shape Modulation for Flapping-Wing Micro-AirVehicle Control During Hover," Journal of Guidance Control and Dynamics, Vol. 33, No. 3, 2010, pp. 724-739.

${ }^{10}$ Faruque, I. and Humbert, J. S., "Dipteran Insect Flight Dynamics. Part 1 Longitudinal Motion about Hover," Journal of Theoretical Biology, Vol. 264, No. 2, 2010, pp. 538-552.

${ }^{11}$ Faruque, I. and Humbert, J. S., "Dipteran Insect Flight Dynamics. Part 2: Lateral-Directional Motion about Hover,” Journal of Theoretical Biology, Vol. 265, No. 3, 2010, pp. 306-313.

${ }^{12}$ Tang, J., Chimakurthi, S., Palacios, R., Cesnik, C. E. S. and Shyy, W., "Computational Fluid-Structure Interaction of a Deformable Flapping Wing for Micro Air Vehicle Applications," AIAA-2008-0615, 46th AIAA Aerospace Sciences Meeting and Exhibit, Reno, NV, Jan. 7-10, 2008.

${ }^{13}$ Chimakurthi, S. K., Stanford, B. K., Cesnik, C. E. S. and Shyy, W., "Flapping Wing CFD/CSD Aeroelastic Formulation Based on a Corotational Shell Finite Element," AIAA-2009-2412, 50th AIAA/ASME/ASCE/AHS/ASC Structures, Structural Dynamics, and Materials Conference, Palm Springs, CA, May 4-7, 2009.

${ }^{14}$ Chimakurthi, S. K., Tang, J., Palacios, R., Cesnik, C. E. S. and Shyy, W., "Computational Aeroelasticity Framework for Analyzing Flapping Wing Micro Air Vehicles,” AIAA Journal, Vol. 47, No. 8, 2009, pp. 1865-1878.

${ }^{15}$ Aono, H., Chimakurthi, S. K., Cesnik, C. E. S., Liu, H. and Shyy, W., "Computational Modeling of Spanwise Flexibility Effects on Flapping Wing Aerodynamics," AIAA-2009-1270, 47th AIAA Aerospace Sciences Meeting Including the New Horizons Forum and Aerospace Exposition, Orlando, FL, Jan. 5-8, 2009.

${ }^{16}$ Heathcote, S., Wang, Z. and Gursul, I., "Effect of Spanwise Flexibility on Flapping Wing Propulsion," Journal of Fluids and Structures, Vol. 24, No. 2, 2008, pp. 183-199.

${ }^{17}$ Cesnik, C. E. S. and Brown, E. L., "Modeling of High Aspect Ratio Active Flexible Wings for Roll Control," AIAA-20021719, 43rd AIAA/ASME/ASCE/AHS/ASC Structures, Structural Dynamics, and Materials Conference, Denver, CO, Apr. 22-25, 2002.

${ }^{18}$ Cesnik, C. E. S. and Brown, E. L., “Active Wing Warping Control of a Joined-Wing Airplane Configuration,” AIAA-20031715, 44th AIAA/ASME/ASCE/AHS/ASC Structures, Structural Dynamics, and Materials Conference, Norfolk, VA, Apr. 7-10, 2003.

${ }^{19}$ Cesnik, C. E. S. and Su, W., "Nonlinear Aeroelastic Modeling and Analysis of Fully Flexible Aircraft," AIAA-2005-2169, 46th AIAA/ASME/ASCE/AHS/ASC Structures, Structural Dynamics, and Materials Conference, Austin, TX, Apr. 18-21, 2005.

${ }^{20}$ Cesnik, C. E. S. and Su, W., "Nonlinear Aeroelastic Behavior of Fully Flexible Slender Vehicles," International Forum on Aeroelasticity and Structural Dynamics 2005, Munich, Germany, Jun. 28 - Jul. 1, 2005.

${ }^{21}$ Shearer, C. M. and Cesnik, C. E. S., "Modified Generalized- $\alpha$ Method for Integrating Governing Equations of Very Flexible Aircraft," AIAA-2006-1747, 47th AIAA / ASME / ASCE / AHS / ASC Structures, Structural Dynamics, and Materials Conference, Newport, RI, May 1-4, 2006.

${ }^{22}$ Shearer, C. M. and Cesnik, C. E. S., "Nonlinear Flight Dynamics of Very Flexible Aircraft," Journal of Aircraft, Vol. 44, No. 5, 2007, pp. 1528-1545.

${ }^{23} \mathrm{Su}$, W. and Cesnik, C. E. S., "Coupled Nonlinear Aeroelastic and Flight Dynamic Simulation of a Flapping Wing Micro Air Vehicle," IFASD-2009-142, International Forum on Aeroelasticity and Structural Dynamics 2009, Seattle, WA, Jun. 21-25, 2009.

${ }^{24} \mathrm{Su}$, W. and Cesnik, C. E. S., "Nonlinear Aeroelastic Simulations of a Flapping Wing Micro Air Vehicle Using Two Unsteady Aerodynamic Formulations," AIAA-2010-2887, 51st AIAA/ASME/ASCE/AHS/ASC Structures, Structural Dynamics, and Materials Conference, Orlando, FL, Apr. 12-15, 2010.

${ }^{25}$ Peters, D. A. and Johnson, M. J., "Finite-State Airloads for Deformable Airfoils on Fixed and Rotating Wings," Proceedings of Symposium on Aeroelasticity and Fluid Structure Interaction Problems, ASME Winter Annual Meeting, edited by P. P. Friedmann and J. C. I. Chang, AD - Vol. 44, The American Society of Mechanical Engineers, New York, NY, 1994, pp. 128.

${ }^{26}$ Peters, D. A., Karunamoorthy, S. and Cao, W.-M., "Finite State Induced Flow Models Part I: Two-Dimensional Thin Airfoil," Journal of Aircraft, Vol. 32, No. 2, 1995, pp. 313-322.

${ }^{27}$ Gao, N., Aono, H. and Liu, H., "A Numerical Analysis of Dynamic Flight Stability of Hawkmoth Hovering," Journal of Biomechanical Science and Engineering, Vol. 4, No. 1, 2009, pp. 105-116.

${ }^{28}$ Sun, M. and Xiong, Y., "Dynamic Flight Stability of a Hovering Bumblebee," Journal of Experimental Biology, Vol. 208, No. 3, 2005, pp. 447-459.

${ }^{29}$ Rosenfeld, N. C. and Wereley, N. M., "Time-Periodic Stability of a Flapping Insect Wing Structure in Hover," Journal of Aircraft, Vol. 46, No. 2, 2009, pp. 450-464.

${ }^{30}$ Amirouche, F. M. L., Computational Methods in Multibody Dynamics, Prentice Hall, Englewood Cliffs, NJ, 1992.

${ }^{31} \mathrm{Su}$, W. and Cesnik, C. E. S., "Nonlinear Aeroelasticity of a Very Flexible Blended-Wing-Body Aircraft," Journal of Aircraft, Vol. 47, No. 5, 2010, pp. 1539-1553.

${ }^{32}$ Richards, J. A., Analysis of Periodically Time-Varying Systems, Springer, New York, NY, 1983.

${ }^{33}$ Friedmann, P. P., "Numerical Methods for Determining the Stability and Response of Periodic Systems with Applications to Helicopter Rotor Dynamics and Aeroelasticity," Computers \& Mathematics with Applications, Vol. 12A, No. 1, 1986, pp. 131148. 